\title{
Anomalous muscles in carpal tunnel associated with neurovascular variations: Case report and brief review
}

\author{
Nabil Eid*, Yuko Ito, Yoshinori Otsuki \\ Department of Anatomy and Cell Biology, Division of Life Sciences, Osaka Medical College, Osaka, Japan; \\ *Corresponding Author: nabileidm@yahoo.com
}

Received 24 October 2013; revised 29 November 2013; accepted 9 December 2013

Copyright (C) 2014 Nabil Eid et al. This is an open access article distributed under the Creative Commons Attribution License, which permits unrestricted use, distribution, and reproduction in any medium, provided the original work is properly cited. In accordance of the Creative Commons Attribution License all Copyrights (C) 2014 are reserved for SCIRP and the owner of the intellectual property Nabil Eid et al. All Copyright (C) 2014 are guarded by law and by SCIRP as a guardian.

\section{ABSTRACT}

Anomalous muscles in carpal tunnel (CT) may cause carpal tunnel syndrome (CTS), and may be also associated with neurovascular abnormalities, altering the diagnosis and surgical interventions for CTS. The authors report a case of bilateral Gantzer's muscles (GMs) inserted into the tendons of flexor digitorum profundus within the CTs in an old male cadaver. The left GM was associated with enlarged lumbrical muscles in the CT and bifid median nerve (MN) communicating with ulnar nerve in the hand. The right GM was associated with perforation of MN branches in the hand by superficial palmar arch. This unreported coexistence of anomalous GMs and the associated neurovascular variations may have clinical relevance to etiology, diagnosis and treatment of CTS.

\section{KEYWORDS}

\section{Carpal Tunnel; Gantzer's Muscle; Median Nerve; Nerve Compression Syndromes}

\section{INTRODUCTION}

Carpal tunnel syndrome (CTS) results from compression of median nerve (MN) inside the carpal tunnel (CT), a narrow fibro-osseous tunnel through which the $\mathrm{MN}$ passes with nine tendons (four flexor digitorum superficialis, four flexor digitorum profundus and flexor pollicis longus). The causes of CTS may be related to pregnancy, systemic diseases or local factors within the CT. Anything that reduces the dimensions of the CT or increases the volume of its contents will predispose to CTS, and many medical associations have been reported, but most cases are idiopathic [1-3]. The anatomy of CT is highly variable, so surgeons should be aware of possible anatomic variations which may affect diagnosis and treatment of CTS. It is not uncommon to find either variations of normally present tendons (palmaris longus, digital flexors, lumbricals) or the presence of anomalous tendons (flexor carpi radialis brevis, palmaris profundus) [4-7]. The accessory heads of the deep flexors of the forearm; GMs have been described as two different small bellies which commonly arise from medial epicondyle and insert into either flexor pollicis longus or flexor digitorum profundus (FDP) and was reported to be involved in compression of anterior interosseous nerve (AIN) [7-11]. Studies regarding the presence of these muscles in the CT are very few. The presence of these accessory muscles in the CT may impact the diagnosis and surgical treatment of CTS.

\section{CASE REPORT}

During cadaveric dissection of 50 upper limbs done for the undergraduate students at the anatomy laboratory, Osaka medical college, we looked for the presence of GMs terminating distally within the CTs. In addition, the CTs were explored for the presence of associated variations. We detected bilateral GMs terminating within the CTs in 73 year-old-Japanese man. The right GM (Figure 1 and upper inset) arose from medial epicondyle deep to flexor digitorum superficialis, coursed distally medial to MN and AIN and entered the CT deep to the TCL where it terminated by joining the tendon of FDP to middle finger. Inside the CT, enlarged lumbrical muscles were detected deep to the $\mathrm{MN}$ which trifurcated into terminal digital branches below the distal edge of TCL. Just distal to the CT, the MN medial branch was abnormally perfo- 
rated by the superficial palmar arch (SPA) (Figure 1 and lower inset). The left GM (Figure 2) originated from medial epicondyle (Figure 2 and lower inset), followed a similar course as the right one and travelled distally deep to the TCL where it was inserted into FDP tendon to the index finger within the CT. There were enlarged lumbrical muscles within the CT close to the termination of GM tendon (Figure 2 and upper inset). Moreover, the MN divided within the CT into two terminal branches (bifid) and there was communication between the medial branch of bifid MN and ulnar nerve just distal to CT.

\section{DISCUSSION}

A few cases of isolated unilateral GM joining the tendon of FDP near the wrist were reported $[9,12]$. One report showed the coexistence of three anatomical variations in the forearm of one cadaver: GMs, Martin-Gruber anastomosis, and nerve of Henle [11]. However, the present study is the first report showing the coexistence in one cadaver of bilateral GMs within CTs, MN perforation by SPA in one side and bifid MN communicating with the ulnar nerve in the other side. The coexistence of these anatomical variations may have clinical relevance to etiology, diagnosis and treatment of CTS and may be important for surgeons and radiologists. The anomalous muscles found in the current case may cause CTS by compressing the $\mathrm{MN}$ via narrowing the space of CT [1-3]. In addition, they may prohibit endoscopic carpal tunnel release $[4,13,14]$. The GMs in our case are not typical as

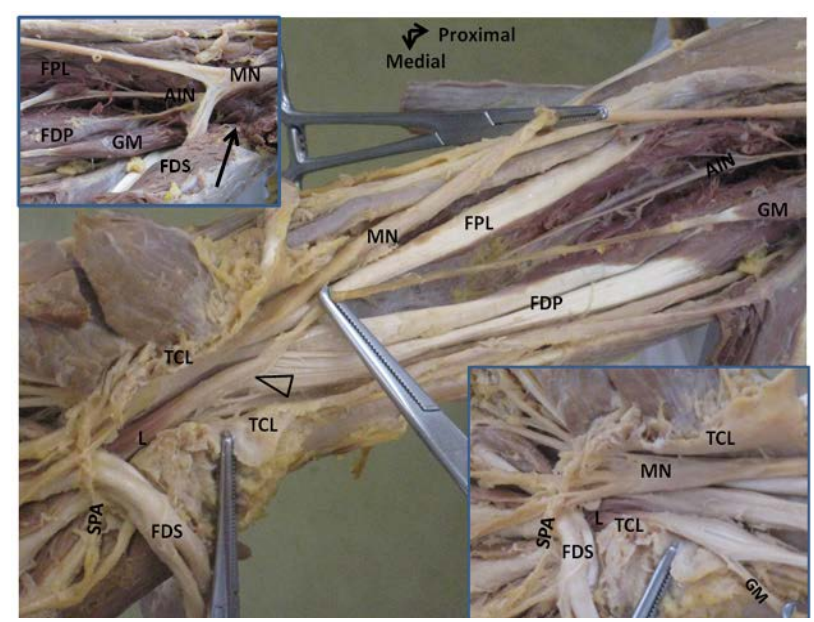

Figure 1. Right GM in carpal tunnel associated with abnormal perforation of MN by superficial palmar arch (SPA). The upper inset shows the proximal part of GM while the lower inset demonstrates the perforation of MN by SPA. GM, Gantzer's muscle; FDP, flexor digitorum profundus; FPL, flexor pollicis longus; MN, median nerve; TCL, transverse carpal ligament; L, lumbrical muscle; FDS, flexor digitorum superficialis; AIN; anterior interosseous nerve; FDS, flexor digitorum superficialis. The open arrow marks the insertion of GM. The arrow in the upper inset indicates the origin of GM.

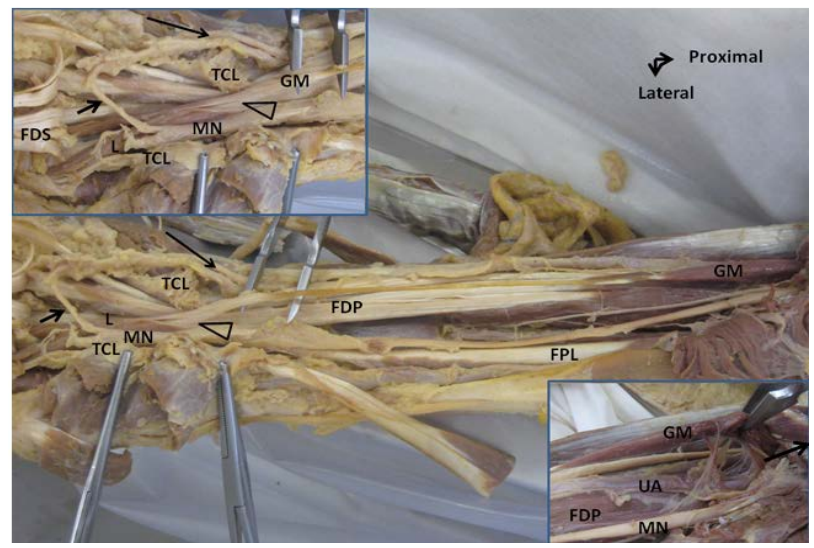

Figure 2. Left GM within carpal tunnel associated with high division of MN. The upper inset shows enlarged lumbrical muscle (L) inside CT and abnormal communication (short arrow) of $\mathrm{MN}$ with the ulnar nerve (long arrow). The lower inset demonstrates proximal part of GM. GM, Gantzer's muscle; FDP, flexor digitorum profundus; FPL, flexor pollicis longus; MN, median nerve; TCL, transverse carpal ligament; L, lumbrical muscle; FDS, flexor digitorum superficialis; UA, ulnar artery. The open arrow head indicates the insertion of GM. The arrow in lower inset indicates the origin of GM.

their tendons joined the tendons of FDP within the CT (they commonly join the tendons of FDP proximal to the CT) [9-12]. The left GM tendon in the present case was inserted into the tendon of FDP to index finger very close to enlarged lumbrical muscles and bifurcated $\mathrm{MN}$ within the CT. The high division of MN (bifid MN) within the CT in association with anomalous muscles has been reported $[15,16]$. Moreover, the medial branch of bifurcated $\mathrm{MN}$ in our case communicated with the ulnar nerve just distal to lower edge of TCL. The communicating branches between the median and ulnar nerves were reported by other researchers and could potentially alter clinical symptoms, signs and electrophysiological studies of CTS resulting in misdiagnosis. For example, sensory disturbance in patients suffering from CTS occurred more frequently in areas innervated by the median nerve (index finger, 94\%) but also with great frequency in other areas (little finger, 39\%) as a result of communication between ulnar and median nerves. Moreover, this communicating nerve may be injured during open CT surgery $[5,17,18]$. In a previous study, we found a case of persistent median artery perforating both the MN and the AIN at the elbow [8]. The right GM in the present case was associated with abnormal perforation of MN terminal branches by the SPA. Because vessels that penetrate nerves have been reported to cause several nerve compression syndromes, there is a possibility of double crush compression of $\mathrm{MN}$ in the current case (within the CT by GM and in the hand by perforating SPA) $[8,19]$. Developmentally, the existence of accessory muscles as GMs connecting the flexor muscles could be explained by the 
incomplete cleavage of the deep layer of the flexor mass, which represents an atavistic character [10].

\section{CONCLUSION}

In conclusion, the presence of anomalous muscles in CTs may be associated with proximal and distal neurovascular abnormalities which should be expected and investigated using ultrasound or MRI for proper treatment of CTS and various hand surgeries.

\section{REFERENCES}

[1] Bland, J.D. (2007) Carpal tunnel syndrome. BMJ, 335, 343-346.

http://dx.doi.org/10.1136/bmj.39282.623553.AD

[2] Luchetti, R. and Amando, P.C. (2007) Carpal tunnel syndrome. Springer-Verlag, Berlin, Heidelberg. http://dx.doi.org/10.1007/978-3-540-49008-1

[3] Demircay, E., Civelek, E., Cansever, T., Kabatas, S. and Yilmaz, C. (2011) Anatomic variations of the median nerve in the carpal tunnel: A brief review of the literature. Turkish Neurosurgery, 21, 388-396.

[4] Jones, D.P. (2006) Bilateral palmaris profundus in association with bifid median nerve as a cause of failed carpal tunnel release. Journal of Hand Surgery (American Volume), 31, 741-743. http://dx.doi.org/10.1016/j.jhsa.2006.02.017

[5] Mhoon, J.T., Juel, V.C. and Hobson-Webb, L.D. (2012) Median nerve ultrasound as a screening tool in carpal tunnel syndrome: Correlation of cross-sectional area measures with electrodiagnostic abnormality. Muscle Nerve, 46, 871-878. http://dx.doi.org/10.1002/mus.23426

[6] Potu, B.K., Gorantla, V.R., Rao, M.S., Bhat, K.M., Vollala, V.R., Pulakunta, T. and Nayak, S.R. (2008) Anomalous origin of the lumbrical muscles: A study on South Indian cadavers. Morphologie, 92, 87-89.

http://dx.doi.org/10.1016/j.morpho.2008.04.006

[7] Eid, N. and Otsuki, Y. (2009) A case of double Gantzer's muscle and its possible role in nerve entrapment. Clinical Anatomy, 22, 881-882. http://dx.doi.org/10.1002/ca.20862

[8] Eid, N., Ito, Y., Shibata, M.A. and Otsuki, Y. (2011) Persistent median artery: Cadaveric study and review of the literature. Clinical Anatomy, 24, 627-633. http://dx.doi.org/10.1002/ca.21127

[9] El Domiaty, M.A., Zoair, M.M. and Sheta, A.A. (2008) The prevalence of accessory heads of the flexor pollicis longus and the flexor digitorum profundus muscles in
Egyptians and their relations to median and anterior interosseous nerves. Folia Morphologica, 67, 63-711.

[10] Jones, M., Abrahams, P.H., Sañudo, J.R. and Campillo, M. (1997) Incidence and morphology of accessory heads of flexor pollicis longus and flexor digitorum profundus (Gantzer's muscles). Journal of Anatomy, 191, 451-455. http://dx.doi.org/10.1046/j.1469-7580.1997.19130451.x

[11] Narayana, K., Narendiran, K., Shetty, K.P. and Prashanthi, N. (2004) A case of coexistence of three anatomical variations in the forearm: Gantzer's muscles, Martin-Gruber anastomosis, and nerve of Henle. European Journal of Anatomy, 8, 81-84.

[12] Vollala, V.R., Nagabhooshana, S., Bhat, S.M., Potu, B.K. and Rakesh, V. (2008) Multiple accessory structures in the upper limb of a single cadaver. Singapore Medical Journal, 49, 254-258.

[13] McClelland Jr., W.B. and Means Jr., K.R. (2012) Palmaris profundus tendon prohibiting endoscopic carpal tunnel release: Case report. Journal of Hand Surgery (American Volume), 37, 695-698. http://dx.doi.org/10.1016/j.jhsa.2012.01.006

[14] Miller, T.T. and Reinus, W.R. (2010) Nerve entrapment syndromes of the elbow, forearm, and wrist. American Journal of Roentgenology, 195, 585-594. http://dx.doi.org/10.2214/AJR.10.4817

[15] Barbe, M., Bradfield, J., Donathan, M. and Elmaleh, J. (2005) Coexistence of multiple anomalies in the carpal tunnel. Clinical Anatomy, 18, 251-259. http://dx.doi.org/10.1002/ca.20086

[16] Pierre-Jerome, C., Smitson, R.D., Shah, R.K., Moncayo, Abdelnoor, V. and Terk, M.R. (2010) MRI of the median nerve and median artery in the carpal tunnel: Prevalence of their anatomical variations and clinical significance. Surgical and Radiologic Anatomy, 32, 315-322. http://dx.doi.org/10.1007/s00276-009-0600-1

[17] Loukas, M., Louis, R.G., Stewar, L.T., Hallner, B., DeLuca, T., Morgan, W., Shah, R. and Mlejnek J. (2007) The surgical anatomy of ulnar and median nerve communications in the palmar surface of the hand. Journal of Neurosurgery, 106, 887-893. http://dx.doi.org/10.3171/jns.2007.106.5.887

[18] Clark, D., Amirfeyz, R., Leslie, I. and Bannister, G. (2011) Often atypical? The distribution of sensory disturbance in carpal tunnel syndrome. Annals of the Royal College of Surgeons of England, 93, 470-473. http://dx.doi.org/10.1308/003588411X586191

[19] Jones, N.F. and Ming, N.L. (1986) Persistent median artery as a cause of pronator syndrome. Journal of Hand Surgery (American Volume), 13, 728-732. http://dx.doi.org/10.1016/S0363-5023(88)80135-7 